BROWN UIIIV PROVIDENCE RI LEFSCHETZ CENTER FOR DYNAMI--ETC F/G 12/1 ASYMPTOTIC BEHAVIOR FOR A SIRONGLY DAMPED NONLINEAR WAVE EQUATI--ETC(U) UNCLASSIFIED JUN BO P MASSATT AFOSR-76-3092

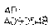
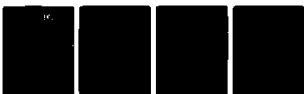

END

EATI

1180

OTIC

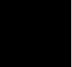

$$
\text { AFOSR-TR-80-1002 }
$$
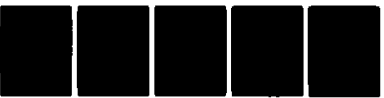

NL
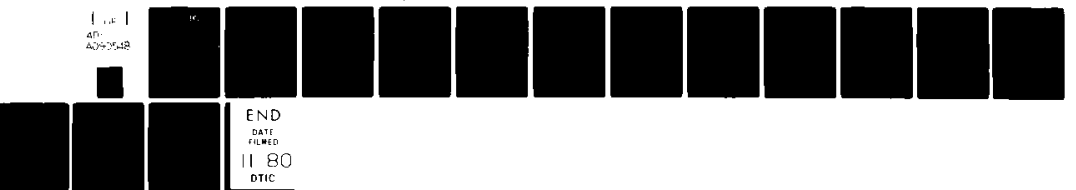


\section{AFOSR-TR- $80-1002$}

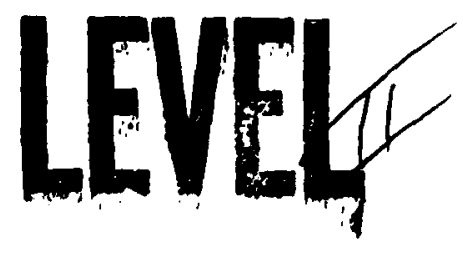

$\infty$
+1
20
0
0
0
0
ASYMPTOTIC BEHAVIOR FOR A STRONGLY DAMPED

NONLINEAR WAVE EQUATION*

by

Paul Massatt ${ }^{* * *}$

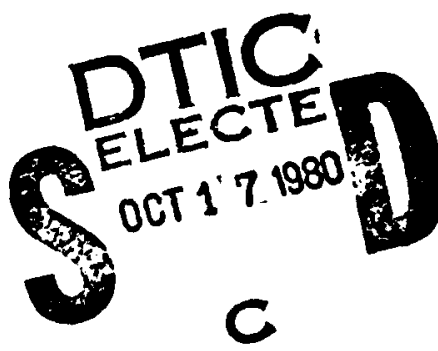

Lefschetz Center for Dynamical Systems

Division of Applied Mathematics

Brown University

Providence, Rhode Island 02912

June 1980

*Invited Lecture, International Conference on Nonlinear Phenomena in Mathematical

Sciences, University of Texas, Arlington, Texas, June 16-20, 1980.

$* * *$

This research was supported in part by Air Force Office of Scientific Research under 1 -AFOSR 76-3092.

$Q \cap+\cap ?$ 


\begin{tabular}{|c|c|}
\hline REPQRT' DOCUMENTATION PAGE & 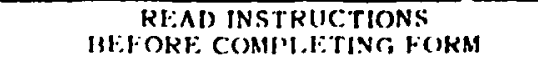 \\
\hline AFOSR TR-3 $10-1002$ AD-A09054 & 3. RECIPIENT'S CATALOG NUMBER \\
\hline ASYMPTOTIC BEHAVIOR FOR A STRONGLY & 5. TYPE OF AEPOAT O PERIOD COVERED \\
\hline DAMPED NONLINEAR & 6. PEAFORMING ThG. RESOAT WUMEER \\
\hline PAUL/MASSATT & 8. CONTAACT OA GRANT NUMBEA (s) \\
\hline $\begin{array}{l}9 \text { PEPFORMING ORGANIZATION NAME AND AODRESS } \\
\text { DIVIS ION OF APPL IED MATHEMATICS } \\
\text { BROWN UN IVERS ITY } \\
\text { PROV IDENCE, RHODE. ISLAND } 02912\end{array}$ & $\begin{array}{l}\text { 10. PROGRAM ELEMENT PROJECT, TASK } \\
\text { AREA O WORK UNIT NUMEERS } \\
61102 \mathrm{~F} / 2304 / \mathrm{A} 1\end{array}$ \\
\hline 1. CONTAOLLING OFFICE NAME AND ADDRESS & 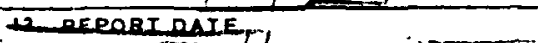 \\
\hline $\begin{array}{l}\text { AIR FORCE OFFICE OF SCIENTIFIC RESEARCH } \\
\text { BOLLING AIR FORCE BASE } \\
\text { WASHINGTON. D.C. }\end{array}$ & Ju NUMEER OF PAGES 12,2 \\
\hline 14. MONITORING AGENCY AME \& ADORESS(if dilferent from Controllina Oflice) & $\begin{array}{l}\text { 15. SECURITY CLASS. (of this report) } \\
\text { UNCLASSIFIED }\end{array}$ \\
\hline & $\begin{array}{l}\text { T5a. DECLASSIFICATION DOWNGRADING } \\
\text { SCHEDULE }\end{array}$ \\
\hline
\end{tabular}

16. DISTRIQUTION STATEMENT (of this Report)

Approved for public release; distribution unlimited.

17. DISTRIBUTION STATEMENT (of the absiract enfered In Block 20. If different (rom Rrport)

18. SUPPLEMENTARY NOTES

19. KEY WORDS (COntinue on reverse side / necessary and identlly by block number)

20. ABSTRACT (Conthute on reverse slde II necossary and identily by block mumber)

- This paper is a specific application of the author's recent paper, on "Limiting Behavior for Strongly Damped Nonlinear Wave Equations" where results of Webb and Fitzgibbon were extend ed by applying results of a few recent papers written by the author. Some of the main results of this paper are to show boundedness of orbits in one space implies boundedness of orbits in other spaces (the technique ehre provides an interesting ... in other spaces (the technique ehre provides an interesting ....

DD, FORM 1473 EDITIONOF I NOV 65 IS OBSOLETE SECUAITY UNCIASSIELED $4018=7$ 
-alternative proof of the main results of Alikakos. Invariant sets in one space are automatically invariant sets in many spaces (which implies smoothness properties of invariant sets), point dissipative and compact dissipative are equivalent in many spaces and imply bounded dissipative in spaces of "smoother" functions, the existence of a "very smooth" maximal compact invariant set under a very weak dissipative assumption, along with its strong stability and attractivity properties in several spaces, and fixed point theorems under these weak dissipative hypotheses of

The first section will show existence, uniqueness and the variation of constants formula, along with certain compactness properties of the orbits for Equation (1). The second dection will discuss boundedness of orbits. The third section will discuss invariant sets. Finally, the fourth section will discuss the limiting behavior under weak dissipative conditions. 


\section{ASYMPTOTIC BEHAVIOR FOR A STRONGLY DAMPED \\ NONLINEAR WAVE EQUATION}

Paul Massatt

In this paper, we consider the limiting behavior of equations of the form

$$
u_{t t}-\alpha \Delta u_{t}-\Delta u=\mathscr{F}\left(t, x, u, \nabla u, u_{t}, \nabla u_{t}\right)
$$

with $\alpha>0$ and $\mathscr{F}$ periodic in $t$. We also consider special cases where $\mathscr{F}$ may depend on fewer terms. We are interested in solutions of the form $u(t, x): \mathbb{R}^{+} \times \Lambda \rightarrow \mathbb{R}$ where $\Lambda$ is a bounded domain in $\mathbb{R}^{\mathrm{n}}$ with smooth boundary, $u+\beta \frac{\partial u}{\partial v}=0$ for all $x \in \partial \Lambda$ and some $\beta \geq 0$, and $u(0, x)=\phi(x)$ and $u_{t}(0, x)=\psi(x)$ for $\phi(x), \psi(x)$ in some appropriate space of initial functions. The case of Neumann boundary conditions could also be considered but is slightly more complicated and so we have omitted this case from discussion in this paper. (See $[14])$.

This paper is a specific application of my recent paper, "Limiting Behavior for Strongly Damped Nonlinear Wave Equations" [14] where results of Webb [16] and Fitzgibbon [4] were extended by applying results of a few recent papers of mine ([11], [12], [13], [14]). I am deeply grateful to Professor Jack K. Hale for his help and supervision in the preparation of this paper.

Before describing the results, a few definitions will be needed. Let $T: X+X$ be a continuous map on a Banach space $X$. A bounded set $B \subset X$ dissipates a set $J$ if there is an integer $n_{0}>0$ such that $T^{n}(J) \subset B$ for all $n \geq n_{0} . T$ is point dissipative if there is a bounded set $B$ which dissipates all points in $X . T$ is compact dissipative if there is a bounded set $B$ which 
dissipates all compact sets in $X, T$ is local dissipative if there is a bounded set $B$ which dissipates a neighborhood of any point in $X . T$ is local compact dissipative if there is a bounded set $B$ which dissipates a neighborhood of any compact set in $X . T$ is bounded dissipative, or ultimately bounded if there is a bounded set $B$ which dissipates all bounded sets in $X$. Some of the main results of this paper are to show boundedness of orbits in one space implies boundedness of orbits in other spaces (the technique here provides an interesting alternative proof of the main results of Alikakos in [1], see [14]), invariant sets in one space are automatically invariant sets in many spaces (which implies smoothness properties of invariant sets), point dissipative and compact dissipative are equivalent in many spaces and imply bounded dissipative in spaces of "smoother" functions, the existence of a "very smooth" maximal compact invariant set under a very weak dissipative assumption, along with its strong stability and attractivity properties in several spaces, and fixed point theorems under these weak dissipative hypotheses.

To understand these results we introduce some definitions. The map $T$ may be thought of as the period map. A fixed point, then, corresponds to a periodic solution. The orbit of a set $B$ under $T, \gamma^{+}(B)$, is defined by $\gamma^{+}(B)=\bigcup_{n=0}^{\alpha} T^{n}(B)$. The $\omega$-limit set of $B, \omega(B)$, is defined by $\omega(B)=\bigcap_{m=0}^{\infty} c 1\left\{\bigcup_{n=m}^{\infty} T^{n}(B)\right\}$. A set $J$ is invariant under $T$ if $T J=J$. Let $B a(x)$ be a ball of radius a centered at $x$. A set $J$ is stable if, for all $\varepsilon>0$, there is a $\delta>0$ such that for all $\mathrm{n} \geq 0, \mathrm{~T}^{\mathrm{n}}\left(\mathrm{J}+\mathrm{B}_{\delta}(0)\right) \subset J+\mathrm{B}_{\varepsilon}(0)$. J attracts $B$ if every neighborhood of $\mathrm{J}$ dissipates B. $J$ is uniformly asymptotically stable if $J$ is stable and attracts a neighborhood of itself.

The first section of the paper will show existence, uniqueness, and the variation of constants formula, along with certain compactness properties of the orbits for Equation (1). The second section will discuss boundedness of orbits. 
The third section will discuss invariant sets. Finally, the fourth section will discuss the limiting behavior under weak dissipative conditions.

1. Existence, Uniqueness, and Compactness Results. Equation (1) may also be considered as an ordinary differential equation on a Banach space. This is the setting I prefer, as it usually seems much more convenient. If $v=u_{t}$, then

$$
\left(\begin{array}{l}
u \\
v
\end{array}\right)_{t}=\left[\begin{array}{ll}
0 & 1 \\
\Delta & \alpha \Delta
\end{array}\right]\left(\begin{array}{l}
u \\
v
\end{array}\right)+\left(\begin{array}{c}
0 \\
\mathscr{F}(t, x, u, \nabla u, v, \nabla v)
\end{array}\right)
$$

or

$$
\begin{aligned}
& z_{t}=-B z+F(t, z) \text { where } B=\left[\begin{array}{cc}
0 & -1 \\
-\Delta & -\alpha \Delta
\end{array}\right], z=\left(\begin{array}{l}
u \\
v
\end{array}\right) \\
& F(t, z)=\left(\begin{array}{c}
0 \\
\mathscr{F}(t, x, u, \nabla u, v, \nabla v)
\end{array}\right) \text {, and } z_{0}=\left(\begin{array}{l}
\phi \\
\psi
\end{array}\right) .
\end{aligned}
$$

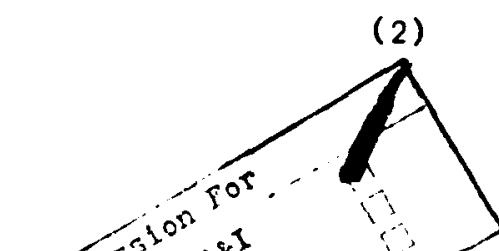

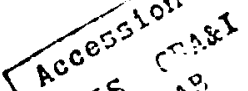
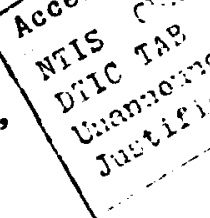

$+a^{2} 0^{3}$

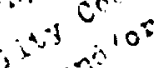

We may consider a variety of spaces, such as $L^{\mathrm{P}} \times L^{\mathrm{P}}, W^{\mathrm{L}}, \mathrm{P} \times \mathrm{L}^{\mathrm{P}}$ (where $W^{\mathrm{m}, \mathrm{P}}$ is the completion in $W^{m, p}$ of $C^{m}$ functions with the appropriate boundary

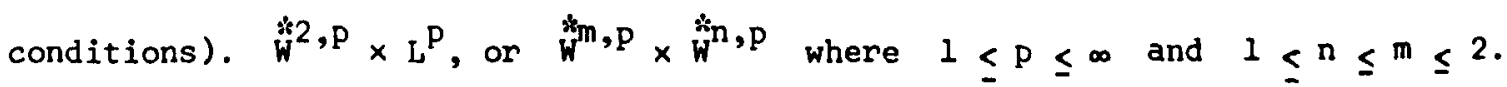

Following the style of D. Henry [9], we show that the solution map to

$$
z_{t}=-B z
$$

sometimes denoted $e^{-B t}$, is an analytic semigroup. An analytic semigroup on a Banach space $x$ is a family of continuous linear operators on $X,\{T(t)\}_{t \geq 0}$ satisfying: 
(i) $T(0)=I, T(t) T(s)=T(t+s)$ for $t, s \geq 0$

(ii) $T(t) x \rightarrow x$ as $t \rightarrow 0^{+}$, for each $x \in X$

(iii) $t \rightarrow T(t) x$ is real analytic on $0<t<\infty$ for each $x \in X$.

The infinitesimal generator $L$ of this semigroup is defined by $L x=\lim _{t \rightarrow 0^{+}} \frac{l}{t}(T(t) x-x)$, its domain $D(L)$ consisting of all $x \in X$ for which this limit exists. We usually write $T(t)=e^{L t}$.

A linear operator $A$ in a Banach space $X$ is called a sectorial operator if it is a closed, densely defined operator such that, for some $\phi$ in $(0, \pi / 2)$ and some $M \geq 1$ and real $a$, the sector $s_{a, \phi}=\{\lambda|\phi<\operatorname{larg}(\lambda-a)| \leq \pi, \lambda \neq a\}$ is in the resolvent set of $A$ and $\|(\lambda-A)^{-1}|| \leq M /|\lambda-a|$ for all $\lambda \in S_{a, \phi^{*}}$

It is known that if $A$ is sectorial, $-A$ generates an analytic semigroup $e^{-A t}$ (see [9]), and conversely, if $-A$ generates an analytic semigroup, then $A$ is sectorial. Furthermore, $e^{-A t}=\frac{1}{2 \pi_{i}^{i}} \int_{\Gamma}(\lambda+A)^{-1} e^{\lambda t} d \lambda$, where $\Gamma$ is a contour in $\rho(-A)$ with arg $\lambda \rightarrow \pm \theta$ as $|\lambda| \rightarrow+\infty$ for some $\theta \in(\pi / 2, \pi)$. If $\operatorname{Re} \sigma(A)>\delta$, then $\left\|e^{-A t} \mid\right\| \leq C e^{-\delta t}$ for all $t>0$ and for some constant $c$. .

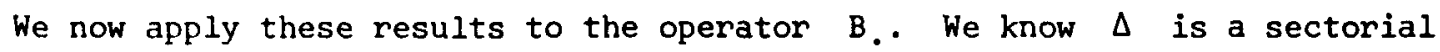
operator on $L^{\mathrm{p}}, W^{\mathrm{l}}, \mathrm{p}$, and $\mathrm{W}^{2}, \mathrm{p}$. The operator $-\Delta$ has simple eigenvalues $\left\{\lambda_{n}\right\}_{n \geq 0}$ with $\lambda_{n}>0,\left\{\lambda_{n}\right\} \rightarrow+\infty$, and corresponding eifenfunctions $\left\{\rho_{n}(x)\right\}$. By using these eigenfunctions we can show $B$ has eigenvalues $\left\{r_{n}\right\} \cup\left\{q_{n}\right\}$ where $r_{n}=\frac{\alpha \lambda_{n}}{2}-\frac{1}{2} \sqrt{\alpha^{2} \lambda_{n}^{2}-4 \lambda_{n}}$ and $q_{n}=\frac{\alpha \lambda_{n}}{2}+\frac{1}{2} \sqrt{\alpha^{2} \lambda_{n}^{2}-4 \lambda_{n}}$. We get $\lim _{n \rightarrow \infty}\left\{r_{n}\right\}=\frac{2}{\alpha}$ and $\lim _{n \rightarrow \infty}\left\{q_{n}\right\}=+\infty$. Hence, we know $\left\{r_{n}\right\} \cup\left\{q_{n}\right\} \cup\left\{\frac{2}{\alpha}\right\}$ is in the spectrum of $B$, with $\left\{r_{n}\right\} \cup\left\{q_{n}\right\}$ in the point spectrum and $\left\{\frac{2}{\alpha}\right\}$ in the continuous spectrum.

We next show that this is the entire spectrum of $B$, and that $B$ is sectorial. To do this we formally calculate the resolvent of $-B,(\lambda+B)^{-1}$. 
We get

$$
(\lambda+B)^{-1}=\left(\lambda^{2}-(\alpha \lambda+1) \Delta\right)^{-1}\left[\begin{array}{cc}
\lambda-\alpha \Delta & 1 \\
\Delta & \lambda
\end{array}\right] .
$$

Since all the operators commute, it is easy to see that the inverse exists for all $\lambda \notin\left\{r_{n}\right\} \cup\left\{q_{n}\right\} \cup\left\{\frac{2}{\alpha}\right\}$. Furthermore, by using the bounds on the resolvent $(\lambda-\Delta)^{-1}$ (since we know $A$ is sectorial) it is very easy to show $B$ is also sectorial. Hence, $e^{-B t}$ is an analytic semigroup. Since $\operatorname{Re} \sigma(B)<0$ we have $\left\|e^{-B t}\right\| \leq K e^{-\delta t}$ for some $K, \delta>0$.

In the following we will use the fact that $e^{-B t}=\int_{\Gamma}(\lambda+B)^{-1} e^{\lambda t} d \lambda$ where $\Gamma$ is a contour in $\rho(-B)$, the resolvent of $-B$, with $\arg \lambda \rightarrow \pm \theta$ for some $\theta \in(\pi / 2, \pi)$. This tells us that $e^{-B t}(v(x))$, considered as an operator on $v(x)$ maps bounded sets in $L^{P}$ to bounded sets in ${ }_{W}^{2}, P \times *_{W}^{2}, P$.

We now return to equation (2). If $F(t, z)$ is Hölder continuous in $t$ and $F(t, \cdot): X+Y$ is locally lipschitz with $D(A)_{X} \in Y(D(A)$ on the space $X$ is continuously imbedded into $Y$ ) then, following D. Henry [9], we have existence, uniqueness, and the variation of constants formula,

$$
z(t)=e^{-B\left(t-t_{0}\right)} z_{0}+\int_{t_{0}}^{t} e^{-B(t-s)} F(s, z(s)) d s .
$$

This formula will constantly be used as we discuss further results.

For compactness properties, it is useful to use the notion of the Kuratowski measure of noncompactness, or $\alpha$-measure. The $\alpha$-measure is a map $\alpha: G \rightarrow[0, \infty]$ where $\rightarrow$ is the collection of bounded sets in $x$ with the property $\alpha(B)=\inf \{r / B$ can be covered by a finite collection of sets of diameter less than $r$ \}. The $\alpha$-measure of noncompactness has the following properties: 


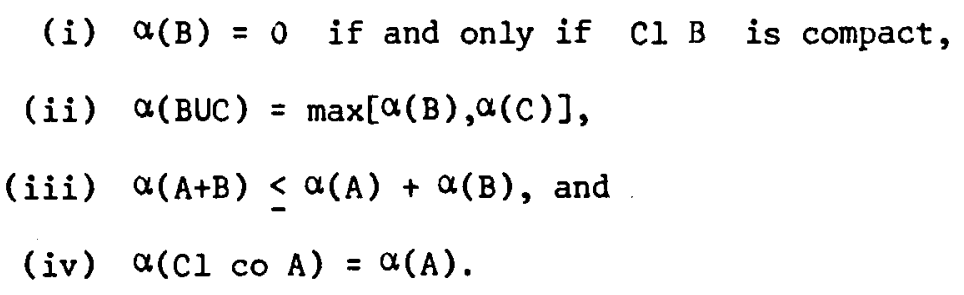

$T$ is called an $\alpha$-constraction if there exists a $k \in[0,1)$ such that for all $B \in \mathscr{S}$ we have $\alpha(\mathrm{TB}) \leq k \alpha(\mathrm{B})$. Often in dynamical systems, we need to consider a more general class of operators, the weak $\alpha$-contraction. $T$ is a conditional $\alpha$-contraction if there exists a $k \in[0,1)$ such that for all B with $\mathrm{TB} \in \mathscr{D}$ we have $\alpha(\mathrm{TB}) \leq k \alpha(\mathrm{B})$. Similarly, $\mathrm{T}$ is conditionally completely cortinuous if for all $\mathrm{B} \in \mathscr{D}$ with $\mathrm{TB} \in \mathscr{D}$ we have $\mathrm{TB}$ is precompact. In all the spaces we have considered, and with $\mathscr{F}$ satisfying the conditions to be mentioned below, the operator $U\left(t, t_{0}\right)$ defined by $U\left(t, t_{0}\right) z_{0}=\int_{t_{0}}^{t} e^{-B(t-s)} F(s, z(s)) d s$ where $z(s)$ is the solution with initial condition $z\left(t_{0}\right)=z_{0}$, is conditionally completely continuous. This will follow from the fact that either $F(t, \cdot)$ is completely continuous, or that $e^{-B t}\left(\begin{array}{c}0 \\ v(x)\end{array}\right)$ maps bounded sets in $L^{P}$ into

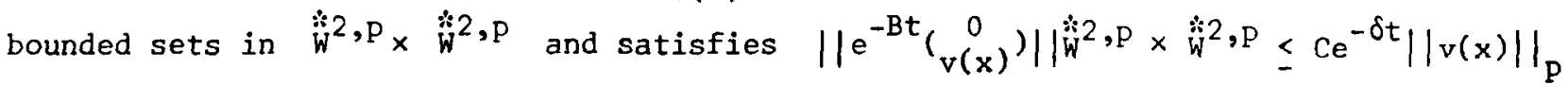
for some $c, \delta>0$. Also, since for each space there is a $c, \delta>0$ such that $\| e^{-B t}|| \leq C e^{-\delta t}$ for any $t>0$ there is an equivalent norm where $e^{-B t}$ is a contraction. Using this and property ( $i i i)$ of the $\alpha$-measure it is easy to see that for all $t>t_{0}$ there is an equivalent norm where $x\left(t, t_{0}\right)$, the solution map of (2), is a conditional $\alpha$-contraction.

Some of the relevance of this is found in the following theorem.

Theorem 1: If $T$ is a conditional $\alpha$-contraction and $\gamma^{+}(B)$ is bounded, then $Y(B)$ is nonempty, compact, and attracts B. Furthermore, if B is precompact, then $\gamma^{+}(B)$ is precompact. 
In order to synthesize the results, we will call $\mathscr{A}$ the class of admissible spaces. Three special classes of admissible spaces will be defined. The class may be enlarged if greater smoothness assumptions on $\mathscr{F}$ are assumed (see [14]).

Case (i): $F(t, z(t))=(\mathscr{F}(t, x, u, \nabla u, v, \nabla v)), \mathscr{F}$ is Hölder continuous in $t$, locally lipschitz and there exists a continuous function $K(\cdot): \mathbb{R}^{+} \rightarrow \mathbb{R}^{+}$such that $|\mathscr{F}(t, x, u, \nabla u, v, \nabla v)| \leq K(t)(1+|u|+|\nabla u|+|v|+|\nabla v|)$. Then the following

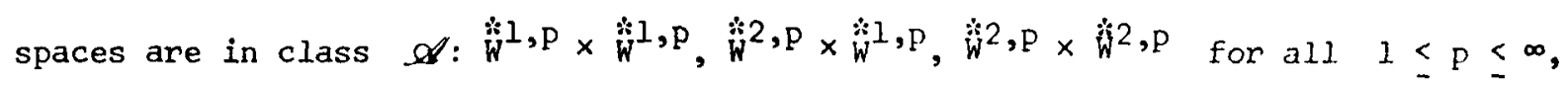
$*^{*} 1, \mu \times *_{c}^{*} 1, \nu$ for $0 \leq \nu<\mu \leq 1, \ddot{*}^{2} \times \stackrel{*}{c}^{1, \nu}$ for $0 \leq \nu<1$, and $\ddot{*}^{2} \times *^{*}$.

Case (ii): $F(t, z(t))=(\mathscr{F}(t, x, u, \nabla u, v), \mathscr{F}$ is Hölder continuous in $t$, locally lipschitz and there exists a continuous function $K(\cdot): \mathbb{R}^{+} \rightarrow \mathbb{R}^{+}$such that $|\mathscr{F}(t, \mathrm{x}, \mathrm{u}, \nabla \mathrm{u}, \mathrm{v})| \leq \mathrm{K}(\mathrm{t})(1+|\mathrm{u}|+|\nabla \mathrm{u}|+|\mathrm{v}|)$. Then the class consists of all the spaces mentioned in (i) and the following additional spaces: $\ddot{W}^{1}, P \times L^{P}, \ddot{W}^{2}, P \times L^{P}, \ddot{C}^{2} \times C$ for $0 \leq \mu<1$, and $\ddot{C}^{1, \mu} \times C^{\nu}$ for $0 \leq \mu, \nu<1$.

Case (iii): $F(t, z(t))=(\mathscr{F}(t, x, u, \nu)), \mathscr{F}$ is Hölder continuous in $t$, locally lipschitz and there exists a continuous function $K(\cdot): \mathbb{R}^{+} \rightarrow \mathbb{R}^{+}$such that $|\mathscr{F}(t, x, u, v)| \leq k(t)(l+|u|+|v|)$.

Then the class consists of all the spaces in (i) and (ii) and the following spaces: $L^{p} \times L^{p}$ for $I \leq P \leq \infty$ and $C^{\mu} \times C^{\nu}$ for $0 \leq \nu \leq \mu<1$.

2. Boundedness of Orbits. It is of ten of great interest to know whether boundedness of orbits in one space implies boundedness of orbits in other spaces. This question was discussed for a predator-prey problem of great generality by Alikakos [1]. The methods discussed here are different, and I believe of a simpler 
nature than those found in Alikakos. The arguments use the fact that the dynamical system is defined on a variety of spaces, the structure of the variation of constants formula, and the repeated application of the Sobolev Imbedding Theorem.

Theorem 2: For Equation (2), if $\gamma^{+}\left(z_{0}\right)$ is a bounded orbit in some admissible space $x \in \mathscr{A}$, then $\gamma^{+}\left(z_{0}\right)$ is a bounded orbit in all admissible spaces $Y$ for that equation provided $z_{0} \in Y$.

The proof may be found in [14]. We give an indication of the proof for an example. Let $z_{0} \in L^{q} \times L^{q}$ and $z(t)$ bounded in $L^{P} \times L^{p}$ with $p<q \leq \infty$. Let $z(t)$ be bounded in $L^{P} \times L^{P} \cdot z(t)=e^{-B\left(t-t_{0}\right)} z_{0}+U\left(t, t_{0}\right) z_{0}$, with $U\left(t, t_{0}\right) z_{0}$ bounded in $\dot{W}^{2}, P \times \ddot{w}^{2}, P$. The Sobolev Imbedding Theorem implies for some $\mathrm{p}$, with $\mathrm{p}<\mathrm{P}_{1} \leq \mathrm{q}$ we have $\dot{\mathrm{w}}^{2}, \mathrm{P} \subset \mathrm{L}^{\mathrm{P}_{1}}$. Hence $\mathrm{U}\left(t, \mathrm{t}_{0}\right) \mathrm{z}_{0}$ is bounded in ${ }_{L}^{P_{1}} \times L^{P_{1}}$, a.d thus, $z(t)$ is bounded in $L^{P_{1}} \times L^{P_{1}}$. Proceeding by induction we can show $z(t)$ is bounded in $\mathrm{L}^{\mathrm{q}} \times \mathrm{L}^{\mathrm{q}}$.

3. Invariant Sets. Here we show an invariant set in one space is a compact invariant set in all the admissible spaces.

Theorem 3: If $\mathrm{J}$ is an invariant set in an admissible space $X$ for the period map $T$, then $J$ is a precompact invariant set in all the admissible spaces for the period map $\mathrm{T}$.

The proof may be found in [14]. Although the proof is similar to the proof of Theorem 2.1, the following lemma is needed to resolve a problem.

Lemma 4. Let $X \subset Y$. Let $\mathrm{J} \subset \mathrm{X}$ be precompact in $X$. Then $\mathrm{Cl} X(\mathrm{~J})=\mathrm{Cl} Y(\mathrm{~J})$ where $C 1 X$ is the closure in the Banach space $x$. 
Now we consider case (iii) of ( 2$)$. Let $J$ be an invariant set in $L^{P} \times{ }_{L}^{P}$. Define $c(n)=e^{-B n \omega}$ and $U(n)=U\left(t_{0}+n \omega, t_{0}\right)$. Then $T^{n}=c(n)+U(n)$. Since $T$ is a conditional $\alpha$-contraction, $J$ is precompact in $L^{p} \times L^{p} . \bigcup^{\infty} U(n) J$ remains bounded in $*^{2}, p \times *^{2}, p$, so for some $p_{1}>p, \bigcup_{n=0}^{\infty} U(n) J$ is precompact in $L^{r} \times L^{r}$ for $p \leq r \leq p_{1}$. But $J=C(n) J+U(n) J$ and $C(n) J \rightarrow 0$ as $n \rightarrow \infty$. Thus, $J \subset C l \times\left(\bigcup_{n=0} U(n) J\right)$. Hence, $J \subset L^{n} \times L^{r}$ by Lemma 4. Proceeding by induction we get $J$ in a precompact invariant set in $L^{q} \times L^{q}$ for all $1 \leq q \leq \infty$. Since $\bigcup^{\infty} U(n) J$ is precompact in $\ddot{W}^{1}, P \times \stackrel{W}{W}^{l}, P$ also, we can get the same result for $\mathrm{n}=0$

$\ddot{W}^{1, q} \times \ddot{W}^{1, q}$ for all $1 \leq q \leq \infty$. Using this we may extend to $*^{2}, q \times *^{2}, q$ for all $1 \leq \mathrm{q} \leq \infty$, and even to $\mathrm{c}^{2} \times \mathrm{c}^{2}$.

4. Limiting Behavior. We will once again follow the format of previous sections. We will state a general theorem, indicate the methods used to prove the result, state several corollaries, and then apply the result to an example.

Theorem 5: Let $X$ and $Y$ be admissible spaces $(X, Y \in \mathscr{A})$ and $Y \subset X$. Suppose a bounded set in $X$ dissipates points in $Y$ under the period map $T$ of (2). Then $T$ is compact dissipative in $Y$. Furthermore, if $Y \subset \subset X$ (compactly imbedded), then $T$ is bounded dissipative in $Y$.

The proof uses the Sobolev imbedding theorem and the following results from $[11],[13]$.

Theorem 6: Let $i: x_{1} \hookrightarrow x_{2}$ be a compact imbedding where $x_{i}$ are Banach spaces topologies denoted $\mathscr{F}_{i}$. Let $T, C$, and $U$ be continuous operators mapping $x_{i}$ into itself, $i=1,2$. Let $T=C+U$ with $C$ a contraction in both spaces and $U$ satisfying the following property: if $B$ and $U(B)$ are bounded subsets 
of $\left(x_{1}, F_{2}\right)$ then $U(B)$ is bounded in $\left(x_{1}, F_{1}\right)$. Let $C(0)=0$. Then point dissipative and compact dissipative in $x_{2}$ are equivalent. Also, the following three statements are equivalent: (i) There is a bounded set in $x_{2}$ which dissipates points in $x_{1}$, (ii) $T$ is point dissipative in $X_{1}$, and (iii) $T$ is bounded dissipative in $x_{1}$.

The following well-known theorem indicates the significance of these results.

Theorem 7: If $T$ is a conditional $\alpha$-contraction and compact dissipative in $X$ then there is a maximal compact invariant set in $X$ which is uniformly asymptotically stable, attracts neighborhoods of compact sets, and has a fixed point (corresponding to a periodic orbit of the equation). If, in addition, $T$ is bounded dissipative, the maximal compact invariant set attracts bounded sets.

We now apply all these results to case (iii) of (2). Let $T$ be point dissipative in $L^{\mathrm{P}} \times \mathrm{L}^{\mathrm{P}}$ (or a weaker condition is to let a bounded set in $L^{r} \times L^{r}$ dissipate points in $L^{p} \times L^{p}$ for $l \leq \gamma \leq p$ ) with $P \leq 1$. Then $T$ is compact dissipative in the following class of spaces, which we will call

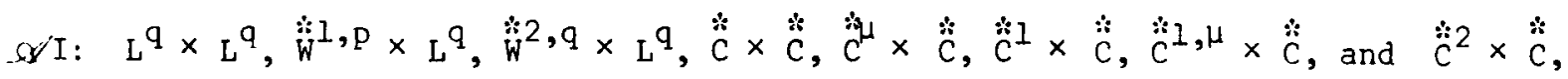
for $\mathrm{p} \leq \mathrm{q} \leq \infty$ and $0<\mu<1$. Also, $\mathrm{T}$ is bounded dissipative in class $\mathscr{A}$ II which consists of the following spaces: $\ddot{w}^{1, q} \times w^{1, q}, w^{2, q} \times w^{1, q}, w^{2, q} \times w^{2, q}, c^{\mu} \times c^{\nu}$, $c^{1} \times c^{\beta}, c^{1, \alpha} \times c^{\beta}, c^{2} \times c^{\beta}, c^{1} \times c^{1}, c^{1, \alpha} \times c^{1}, c^{2} \times c^{1}, c^{1, \mu} \times c^{1, \nu}, c^{2} \times c^{1, \beta}$, and $\ddot{c}^{2} \times \ddot{c}^{2}$, where $q \geq p, 0<\beta, \alpha<1$, and $0<\nu \leq \mu<1$. In addition, there is a set $K$ which is the maximal compact invariant set for each admissible space, and in classes $\mathscr{A}$ and $\mathscr{A I}$ it is uniformly asymptotically stable and attracts neighborhoods of compact sets in class $\mathscr{I}$ and bounded sets in $\mathscr{A}$. Furthermore, $T$ has a fixed point (corresponding to a periodic orbit).

The proofs follow from the fact that an invariant set in one of the spaces must 
be a precompact invariant set in all the spaces. Hence a maximal compact invariant set in one space becomes a maximal compact invariant space in all of the spaces. The attractivity properties follow from Theorem 7 , and the original dissipative results follow from Theorem 6 , the same inductive process, and repeated application of the Sobolev Imbedding Theorem.

In applications, it is often possible to prove boundedness or dissipative properties in some special space; for example, Lyapunov functions are of ten employed. But the same techniques cannot be applied to obtain boundedness or dissipative in any other spaces. The above results allow one to extend the boundedness and dissipative properties to many other spaces, along with the existence, very strong stability and attractivity properties of the maximal compact invariant set.

\section{REFERENCES}

[1] Alikakos, N.D., An application of the invariance principle to reactiondiffusion equations, J. Differential Equations 33(1979), 201-225.

[2] Billotti, J.E. and J.P. LaSalle, Periodic dissipative processes, Bull. Amer. Math. Soc. $6(1971), 1082-1089$.

[3] Cooperman, G.D., $\alpha$ condensing maps and dissipative systems, Ph.D. thesis, Brown University, June 1978.

[4] Fitzgibbon, W.E., Existence theorems for a class of strongly damped quasilinear evolution equations, Dept. of Math., University of Houston, Houston, Texas 77004 to appear.

[5] Hale, J.K., $\alpha$-contractions and differential equations, Proc. Equations Differential Fon. Nonlin. (Brussels 1975) Hermann, Paris, 15-42.

[6] Hale, J.K., Theory of Functional Differential Equations, Appl. Math. Sci., vol. 3, Springer-Verlag, New York, 1977.

[7] Hale, J.K., LaSalle, J.P. and M. Slemrod, Theory of a general class of dissipative processes, J. Math. Ana. Appl. 39(1972), 177-191. 
[8] Hale, J.K. and O. Lopes, Fixed point theorems and dissipative processes, J. Differential Equations 13(1973), 391-402.

[9] Henry, D., "Geometric Theory of Semilinear Parabolic Equations", Lecture Notes in Mathematics, Springer-Verlag. To appear.

[10] Massatt, P.D., Some properties of condensing maps, Annali di Mat. Pura Appl. to appear.

[11] Massatt, P.D., Stability and Fixed Points of Point Dissipative Systems, J. Differential Equations, to appear.

[12] Massatt, P.D., Properties of condensing maps and dissipative systems, Ph.D. thesis, Brown University, June 1980.

[13] Massatt, P.D., Attractivity properties of $\alpha$-contractions Div. of Appl. Math., Brown University, Providence, R.I. 02912, to appear.

[14] Massatt, P.D., Limiting behavior for strongly damped nonlinear wave equations, Div. of Appl. Math., Brown University, Providence, R.I. 02912, to appear.

[15] Webb, G.F., Compactness of bounded trajectories of dynamical systems in infinite dimensional spaces, Dept. of Math., Vanderbilt University, Nashville, Tennessee 37235 , to appear.

[16] Webb, G.F., Existence and asymptotic behavior for a strongly damped nonlinear wave equation, Dept. of Math., Vanderbilt University, Nashville, Tennessee 37235 , to appear. 